\title{
L'économie et la santé vont-elles vraiment de pair?
}

\section{Anna Sax}

Lic. oec. publ., MHA, membre de la rédaction

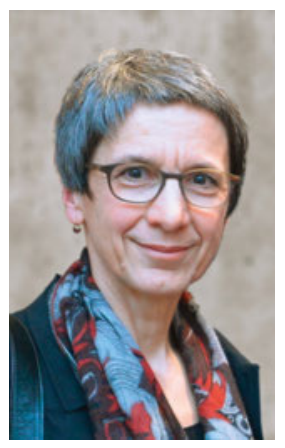

«Mieux vaut être riche et bien portant que pauvre et malade»: c'est ce que nous disions quand nous étions enfants. Mais nous pressentions déjà que cela allait audelà de la simple maxime. Depuis, dans beaucoup de pays, des recherches ont été menées sur les liens entre formation, milieu social et santé. De nos jours, nous percevons la promotion de la santé et la prévention comme des missions interdisciplinaires. L'OMS désigne par l'expression «Health in All Policies» cet engagement qui consiste à contrôler également les décisions politiques à l'aune de leurs conséquences sur la santé publique et l'égalité des chances devant la santé. La politique de la santé couvre donc beaucoup plus que l'organisation et le financement des prestations de santé.

En Suisse, l'espérance de vie d'une personne de 30 ans, diplômée de l'enseignement supérieur, dépasse de quatre ans et demi celle d'un travailleur du même âge sans qualification. Les enquêtes sur la santé montrent que les femmes et les hommes ayant un bon niveau de formation ont en moyenne une meilleure perception subjective de leur santé que les personnes qui ont un bagage de formation léger. Mais comment fonctionnent exactement ces corrélations? Les liens de causalité sont-ils aussi évidents qu'il y paraît à première vue? Ceux qui connaissent un peu les statistiques le savent: même si les personnes ayant des revenus moins élevés souffrent plus souvent de problèmes de santé, cela ne prouve pas que la pauvreté rend malade. Cela montre à la rigueur qu'on a plus de chance d'être bien portant quand on a de l'argent.

Dans ce numéro du Bulletin des médecins suisses, le responsable de la politique de la santé de l'organisation faîtière des entreprises economiesuisse sort une recette étonnamment simpliste pour répondre à cette problématique complexe: plus un pays est riche, plus sa population est en bonne santé, explique-t-il, mettant par conséquent la croissance économique en haut de la liste des priorités de la politique de la santé. Pour Fridolin Marty, une «forte croissance économique empêche même probablement plus de maladies que la plupart des actions classiques de prévention». C'est une thèse bien audacieuse pour un économiste qui ne s'est pas distingué jusqu'à présent comme un expert de la prévention. On ne peut reprocher à F. Marty de n'être pas conscient de l'importance de la formation pour la santé; on peut lire de sa main aussi que la politique de la formation est essentielle à l'égalité des chances, donc à la santé. Cette argumentation raccourcie, reliant directement l'amélioration de la santé à la croissance économique, n'en est que plus étonnante.

F. Marty cite la Grèce comme exemple, pour illustrer qu'une économie en berne entraîne des restrictions budgétaires dans le secteur de la santé. Certes, suite à la crise financière de 2008, la Grèce a connu une chute dramatique des soins de santé, ce qui a entraîné de graves dommages pour la santé de la population. Le graphique de F. Marty montre que les dépenses de santé ont affiché un pourcentage de baisse beaucoup plus accentué que l'ensemble de l'économie. Les mesures d'économie du gouvernement grec n'ont épargné ni la formation, ni la santé. Il existe d'autres exemples de pays qui ont été fortement touchés par la crise de l'euro, et qui n'ont cependant fait aucunes coupes dans la santé; entre 2007 et 2015, l'Espagne, entre autres, a pu augmenter de 7,8\% à 9,2\% la proportion de son produit intérieur brut consacrée aux dépenses de santé. Malgré une situation économique beaucoup plus fragile, les Espagnoles et les Espagnols ont, avec les Suisses, l'espérance de vie la plus élevée. En revanche, les indicateurs de santé des Etats-Unis, l'un des pays les plus riches du monde, sont loin d'être bons.

La promotion de l'économie n'est pas encore une stratégie de santé publique et la croissance économique n'aboutit pas à l'égalité des chances sans mesures complémentaires. Au sens d'economiesuisse, la promotion de l'économie signifie «amélioration des conditions pour l'économie», donc moins d'impôts, moins de charges sociales, moins d'obligations concernant l'environnement et des conditions d'emploi plus souples. Il se pourrait que «l'économie» en profite (quelle que soit la réalité que couvre ce concept, personnes ou objet). Mais la population, elle, n'en tirera aucun profit si, en même temps, on retire au secteur public des moyens qui pourraient être investis dans la formation et la sécurité sociale. 